gastric trouble, usually situated about the lesser curvature, of irregular shape, and with hard eäges, does not concern our present remarks. The so-called acute ulcer, which is comparatively rare, is of great interest in the present connexion. It is situated on a line drawn along the stomach from the centre of the cardiac to the centre of the pyloric orifice-i.e., farthest from the main vessels of supply. Unlike the chronic ulcer it is strikingly circular in shape. Its edges are not infiltrated or hard and examination of recently perforated specimens shows that the edges of the perforation are com posed of gangrenous tissue. A frequent history noted by all observers is that serious stomach symptoms have not, as a rule, preceded the attack. Another point frequently observed and which our view only, we think, explains, is that a second ulcer may be found on the posterior wall at a spot opposite that on the anterior.

We are indebted to Dr. J. Collingwood Stewart, late resident house physician at the Royal Infirmary, for an answer to certain questions which we considered might be usefully inquired into in the history of cases of gastric ulcer. The first was as to symptoms which might be caused by attacks of acute Jistension of the stomach. He ascertained that, with few exceptions, patients in whom a diagnosis of gastric ulcer had been made were subject to attacks of epigastric pain with a feeling of distension, accompanied by breathlessness, sufficient to necessitate loosening corsets or tight bands. In all cases where the diagnosis was verified by operation these symptoms were present.

We thought that excess of hydrochloric acid in the stomach might be accounted for by an extra consumption of sodium chloride and the second question referred to the liking for salt. Dr. Stewart's inquiry showed that out of 40 chlorotic girls more than one-half (25) took an unusual quantity of salt. Of 30 patients with symptoms of gastric ulcer three-fifths liked salt in excess. One "carried a piece constantly"; another "took a pinch whenever she got a chance."

Our view briefly is that excess of hydrochloric acid in the stomach, by producing pyloric spasm and acute gastric distension in an anæmic girl, gives rise to a small gangrenous patch or patches in the stomach wall, and that subsequent digestion of the dead or devitalised patch, if all the coats are involved, leads to perforation. It is generally recognised that the stomach juices in certain circumstances destroy its own wall. We may add that a sudden attack of distension due to pyloric spasm in the case of a chronic ulcer probably accounts for perforation in many instances.

Newcastle-upon-Tyne.

\section{THE RESULTS OF OPERATION FOR RADICAL CURE OF HERNIA. ${ }^{+}$}

\section{BY J. HUTCHINSON, JUN., F.R.C.S. Eng.,} SURGEOY TO THE LONDOX HOSPITAT.

THE aim of this paper is to test in the most stringent manner possible the results of an operation-that for radical cure of hernia-which now occupies a large proportion of the surgeon's time and energy. I find, for example, that over 500 out of a total of 4500 operations which I have performed in the London Hospital during the last 15 years have been done on hernix. Thus it is probable that at least one-tenth of the general run of surgical operations in our hospital work is directed to obtaining the radical cure of hernia. The proportion of the different forms may be roughly estimated from my own record: inguinal hernia, 360 ; femoral hernia, 100 ; and umbilical, ventral, \&c., herniæ, 50. These include about 150 cases of strangulated hernia. The answer to the question, - In what proportion is a permanent and complete cure obtained? - has been a varied one. As Mr. John Langton wrote in $1899^{2}$ : "Afterwards ...... is after all the crucial test of our operative work; at present this 'afterwards ' too often lies buried in the depths of the great unknown from causes over which we have but little control." Mr. Langton limited his consideration to cases of inguinal hernia in private practice and concluded, without giving exact statistics, that " in properly selected cases cures will result

1 A paper read before the Melical Society of London on March 26th, 1906.

3 Transactions of the Medical Society of London, 1899, p. 204. temporarily, at any rate, in about 80 per cent., but it is probable that relapses are not infrequent."

I would urge that hospital patients afford a better test of the operation for radical cure than do private ones, granting that it is more difficult to follow up those belonging to the former class. It is in those who are engaged in hard manual labour-in stokers, soldiers, railway porters, and the likethat the result of our procedure is best estimated. Moreover, certain conditions must be thoroughly applied, especially with regard to the length of time that has elapsed since the operation. Bassini, to whom surgeons are indebted for having shown the importance of opening up the inguinal canal in the great majority of cases, reported cures in no less than 96 per cent. But his limit of time-nine months or over-was certainly too short and to some extent invalidates his statistics. I have taken as the shortest limit two years from the operation, but so many of my cases have been examined seven, eight, or even ten years afterwards that the average works out at about six years. This will probably be accepted as sufficient. If a man has engaged in his ordinary work for six years after the operation and shows not the smallest sign of recurrence the term "radical cure" may fairly be applied. Again, differing from some authorities, reject the use of any truss after the operation, since the patient has submitted to it, as a rule, in order to dispense with the inconvenience of wearing one. In four or five of the cases included in my list the patient has of his own choice worn a truss at some time or other after the operation but not on account of recurrence. In all cases of umbilical hernia after operation an abdominal belt should be worn; in a few examples of inguinal hernia (especially the direct form) and of femoral hernia in elderly people a truss is ordered after the operation. But in the statistics that follow it will be understood that these conditions have been carefully observed : (1) that at least two years, and on an average from five to six years, have elapsed before the result is tested; (2) that no truss has been ordered to prevent recurrence and that within six weeks of the operation (often less) the patient has been directed to return to his work; (3) that the smallest tendency to bulge at the site of operation has been considered as a recurrence of the hernia; and (4) that any development of a hernia at another site has been recorded. The last point will be found to be of considerable importance, though it is curiously omitted by nearly all the writers on the snbject.

The result of operation for radical oure on inguinal hernia.Formerly it was considered inadvisable to operate on hernial subjects (apart from strangulation) who were over 40 years of age. But of late years this limit has been extended and in my list six patients were 50 years old or over. The best results of all are certainly to be obtained on young children, but it happens that only four of the cases in my list were under the age of five years at the time of operation. The great majority were men between 20 and 40 years of age. I have followed up 109 patients after operation on inguinal hernia with the limit of at least two years. In four of these the herniæ were double, making a total of 113. 13 were cases of strangulation (with five recurrences) and it seems fair to leave these out since several were patients of very advanced age, three being 55 years old, one 65 years, and one 74 years. Out of 100 cases primarily done for radical cure, eight of the herniæ have relapsed in situ; of these in five the recurrence was pronounced, one very slight, and two doubtful. Seven patients have since the operation developed an inguinal hernia on the opposite side and one a femoral hernia. I must admit that this result, 8 per cent. or less of true recurrence, is somewhat better than $I$ had expected.

After all, the formation of a hernia elsewhere does not detract from the original operation being termed a radical cure. And if, judged from the most severe standpoint, the proportion of relapses is only from 6 to 10 per cent., the use of the words "radical cure" is thoroughly justified. The statistics of several surgeons relating to Bassini's method practically agree with my own. Thus Carlé and Nicoladoni found 6 per cent. of relapses in situ, and Ceccopieri and Scarrone 7 per cent. One may be allowed some suspicion of the much more favourable results that are now and then brought forward. Thus Lucas-Championnière claims to have bad only 4 per cent. of recurrence but in one of his tables it will be noticed that only half the total number were followed up for more than two months! This fact, to my mind, renders valueless his statistics, since recurrence, if it does follow, usually occurs from nine to 18 months after the operation, There is, of course, no infallible limit and of the eight caser 
of recurrence in my list one developed two years and two others three years after the operation; until that time had elapsed the cure appeared to be perfect. Some of my cases were operated on 15 years ago and there is no doubt that improved methods during this time have diminished the proportion of recurrence. Mr. Langton quotes Tilanus's statistics in 1879 of only 10 per cent. of cures and Socin's of only 40 per cent. It is, however, surprising to find Bull, in a fairly recent paper, stating that 60 per cent. of his cases ultimately relapsed. There must surely have been some grave fault in the form of operation.

Before considering the question of the relative value of the different methods now employed I may allude to one satisfactory fact that came out in the investigation of the cured cases. Practically without exception these patients stated that their operation scars never gave them the slightest trouble and many expressed themselves as improved in general condition and vigour by the removal of the hernial protrusion.

The best methods of radioal cure. -I believe that on only two points are surgeons unanimous with regard to the details of radical cure-first, that the incision should be made directly over the inguinal canal, and secondly, that the hernial sac should be separated from the cord as high as the internal ring and there closed by ligature. But with regard to the next question-Should the canal be opened up by division of the external oblique ?-much diversity of opinion has been expressed. Some (for example, Mr. Edmund Owen ${ }^{3}$ and $\mathrm{Mr}$. T. H. Kellock ${ }^{4}$ ) express some fear of weakening the canal by this cause and urge that a cure can be obtained without it. This may fairly be admitted in the case of young boys, though even in them it is easier to deal thoroughly with the sac after the aponeurosis has been divided. I believe, with the majority of writers on the subject, that in order to narrow the canal it is advisable first to open it up as high as the internal ring and that careful suturing of the aponeurosis will leave it as strong as, or even stronger than, it was before. I have met with one case and one only in which the whole anterior wall of the canal bulged after such suturing (with kangaroo tendon). Primary union had occurred and I cannot account for this solitary exception. In the 100 cases quoted the aponeurosis was divided in almost all.

With regard to the method of dealing with the sac two chief opinions are held. Many surgeons simply tie the sac at the internal ring and let the stump retract; others twist and fix the proximal portion in a new position. I am a firm believer in the latter method. If the sac is of average thickness it is brought through a small opening towards the anterior superior spine and secured there (thoroughly twisted up) by a double tendon suture which transfixes the muscles and the sac. I have never met with a single case of recurrence at the spot where the twisted sac had been brought through the abdominal wall in this manner. If, however, as in some large old hernix, the sac is very thick I tie it with a Staffordshire knot high up and pass both ends of the ligature on a mounted needle through the muscular wall near the anterior superior spine, subsequently knotting the ends tightly together so as to displace the stump upwards and outwards well under the thick muscles in this position. Sir William Macewen's plan of folding up the sac in the subperitoneal space has, I believe, gone out of use, and it appears to have little to recommend it.

Narrowing the inguinal canal.-The late Professor J. Wood was the true pioneer in all that relates to radical cure of hernia, and his contention that it was necessary to restore the valvular action of the conjoined tendon as well as to deal with the sac has been abundantly proved. It is shown especially by the greatly improved results that have been obtained since Bassini introduced the open method of suturing the conjoined tendon, behind the cord, to Poupart's ligament. For practical purposes the methods of narrowing the canal are only three: (1) Its complete obliteration between the external and internal rings behind the cord (Halsted); (2) narrowing it in front of the cord by sewing down the internal oblique and transversalis (Professor Wood, Sir William Macewen, and others); and (3) narrowing it behind the cord (Bassini). The second method is sometimes wrongly described as obliteration of the canal. All three methods are good, though not one of them absolutely guarantees the patient against all chance of recurrence.
In cases which have been previously operated on, in inguinal hernia in women, and in cases of retained testis where the organ cannot be brought down to the scrotum, the complete obliteration of the canal should be practised. There are some others in which the conjoined tendon is poorly developed and cannot be sutured to Poupart's ligament without undue strain-for these also Halsted's method is adrisable.

Of the other two, Bassini's plan-narrowing the canal posteriorly - is probably the most efficient, and it has the endorsement of many surgeons. Of my 100 cases the majority were operated on by Bassini's method; in the earlier ones no formal narrowing of the canal was practised, whilst in about 20 either the canal was obliterated or its anterior wall was tightened up. Of the eight cases of recurrence Bassini's method has been employed in two, Macewen's in one, whilst in five the sack had been twisted and displaced without formally narrowing the canal. This, I think, is conclusive evidence that in adults thorough dealing with the sac alone does not suffice. It should be noted that one patient with poorly developed muscles had a double hernia and recurrence followed operation on both sides, counting as two cases. Another, a case of double direct hernia, recurred on one side only after Bassini's operation had been done on both. A third "patient proved to be so unruly and violent after the operation that recurrence of the hernia was only to be expected. Two cases are included as doubtful recurrence; in them no bulging could be made out on examination but the patients stated that it sometimes was present.

It has been urged against narrowing or obliteration of the inguinal canal that orchitis, varicocele, and hydrocele frequently result. In my own cases I could not ascertain that any one of these complications had happened. In one patient the testis was hard and lumpy, but reference to the notes proved that it was in this condition before the operation. In another a slight varicocele was present. When the canal is so narrowed as to injure the vessels of the cord there is undoubtedly a risk of subsequent orchitis or even atrophy of the testis. But I think this can be prevented by observing the effect of the suturing at the time of operation on the circulation in the cord. And unless there is a true varicocele present it is both unnecessary and inadvisable to excise any of the vessels of the cord. In former years when surgeons were given to suturing the pillars of the external ring closely together these testicular complications were frequent, and their free occurrence after the use of silver wire (Bloodgood and Halsted ${ }^{5}$ ) will be alluded to later.

The material for the deep sutures. - If it be taken as proved that narrowing or obliteration of the canal is advisable in the great majority of cases it follows that the use of deep or buried sutures is a necessity. Although sterilised silk is still employed by a large number of operators I have no hesitation in expressing $\mathrm{my}$ preference for kangaroo tendon. $\mathrm{Mr}$. Langton, -Mr. G. R. Turner, and others have written in its favour. My own experience of kangaroo tendon goes back to 1890 , since which time I have buried in the patients' tissues many thousands of tendon sutures. Unlike silk they hardly ever work out and they can be kept in perfect condition for years in an alcoholic solution of carbolic acid; no material is at once so strong, supple, and non-irritating. It is a delusion to think that they are absorbed in a month or two for I have had occasion to examine such tendon sutures two and three years after I had inserted them. Whilst blending with the surrounding fibrous tissue to some extent they can still be recognised at the end of this time. The fact is the tendon is not absorbed but becomes part of the living tissues. Like every other surgeon who has used kangaroo tendon much, I have known a few cases in which these buried sutures have caused suppuration, but as Mr. Langton points out, the trouble is soon over, whereas buried silk or silkworm-gut may sometimes worry the patient with sinuses for months. If the tendon does irritate it has probably been over-prepared with biniodide of mercury or some other unsuitable antiseptic. It is most important just before the operation to transfer it from the alcohol to sterile cold water, in which it should lie for 20 minutes or so. I cannot understand any operator who has given a thorough trial to the tendon for buried sutures ever being afraid of inserting them or of wishing to change to any other material. Professor Wood used kangaroo tendon in many of his cases and silver wire (which he subsequently removed) in others. Of late years some American surgeons have advised 
silver wire for all the sutures, buried and superficial. In Mr. Bloodgood's voluminous work on hernia already mentioned, ${ }^{5}$ a long series of cases are given in which the canal was narrowed or obliterated by silver wire; in some of them the rectus muscle was dragged down to Poupart's ligament by means of it. The silver wire is supposed by the writer to exert an antiseptic action on the tissues, an action which can only be superfluous if asepsis be obtained. To my mind no region of the body is less suitable for burying silver wire than the groin and its drawbacks were experienced by Mr. C. B. Lockwood, ${ }^{7}$ who tried the method in 15 cases with dismal results. In Bloodgood's cases, whatever was the result as regards recurrence of the hernia, the number of troublesome complications recorded is most remarkable. Induration of the epididymis was even referred to as being usual (p. 428) and orchitis followed by atrophy of the testis was frequent. Yet the result in the latter cases was termed "perfect" (p. 480 and elsewhere). Hæmatoma of the scrotum, sloughing of part of Poupart's ligament (p. 427), cedema of the lower limb from obstruction of the femora vein (three cases), and other complications which could be quoted make up a formidable list which should deter surgeons from using these rigid wire sutures in the radical cure of hernia.

The operation on femoral hernia.-I have only succeeded in following up 16 cases for more than two years, 14 women and two men. The average period at which they were last traced was four and a half years after operation, the average age of the patients at the time being 42 years. There were two recurrences-i.e., 12 per cent. In one of these the recurrence took place three years after operation. In the other case the patient was nearly 60 years of age and had chronic bronchitis. She would not have been operated on for radical cure but for the fact that the hernia was strangulated. Although the number of cases is not large it bears out the statement of Mr. Bloodgood ${ }^{8}$ that "the cure of a femoral hernia is not a difficult problem."

I have occasionally turned up a flap of pectineus muscle to close the ring, but in the great majority of cases $I$ have twisted and fixed the neck of the sac high up with kangaroo tendon and then sutured with the same material Poupart's ligament to the deep fascia, using two or three separate buried sutures. One has to be careful to avoid injurious pressure on the femoral vein ; in one case there was evidence of this, the patient's leg swelling from time to time. In one case of strangulated femoral hernia interesting evidence was obtained of the efficiency of the method alluded to. The patient was an old woman who was suffering from bronchitis, from which she died four days after the operation. At the post-mortem examination $I$ found the femoral ring to be well closed ; it resisted firm pressure from above made with the finger.

I have had no personal experience with Roux's method of radical cure-fixation of Poupart's ligament to the pubis by means of a metal staple. Mr. J. Crawford Renton ${ }^{9}$ records 25 cases of its use and speaks highly of it. Roux is said to have operated 136 times in this way with only two recurrences of the hernia. The objections that might be raised-viz., that the staple would be liable to work loose and that the femoral vein might be compressed too much-are presumably without much ground. The method seems especially indicated where the femoral ring is widely patent.

One point in the after-treatment of radical cure may here be alluded to-the length of time requisite to secure a sound scar. In both inguinal and femoral cases the wound is, as a rule, soundly healed at the end of a fortnight or three weeks. Is it necessary to insist on the patient resting for more than this time? Some operators, I believe, require two or three months' abstention from work on the part of thei patients. But with working men this is a very serious consideration and may mean the entire loss of their previous employment. I see no reason for believing that such prolonged rest is required and have therefore never advised it.

In conclusion, with regard to the radical cure of inguinal hernia the following observations may be made: (1) In all but the simplest cases in children it is best to open up the canal and to narrow it by deep sutures; (2) suturing the conjoined tendon to Poupart's ligament behind the cord by a series of interrupted sutures is probably the best method of

6 Bloodgood and Halsted : Johns Hopkins Reports, 1900. 7 Lockwood: Transactions of the Medical Society of London, 1905 p. 168 .

Johns Hopkins Reports, 1900, p. 365.

9 Contributions to Clinical Surgery, p. 140. narrowing the canal; (3) where the conjoined tendon is deficient, in all recurrent cases, and in inguinal hernia in women the canal should be obliterated ; (4) kangaroo tendon is admirably adapted for the suture material, silver wire being probably the worst; (5) recurrence of the hernia in situ may possibly take place at any length of time after the operation, but if two years be adopted as the limit it should not occur in more than from 5 to 8 per cent.; and ( 6 ) the development of a hernia at another site after operation may be expected in a proportion of cases at least as large as that ust given.

Park-crescent, W.

\section{ON THE TREATMENT OF CANCER BY THERAPEUTIC INOCULATIONS OF A BACTERIAL VACCINE,}

CONTROLLED BY MEASUREMENTS OF THE OPSONIC POWER OF THE BLOOD. ${ }^{1}$

BY DR. C. JACOBS,

AGRÉGÉ AT THE FACULTY OF MEDICINE OF BRUSSELS; $\mathrm{AND}$

DR. VICTOR GEETS,

ASSISTAN'T AT THE INSTITUT ST, ANNE; SOMETIME DEMONSTRATOR OF' PHYSIOLOGY IN THE PHYSIOLOGICAL LABORATORY

OF THE UNIVERSITY OF LONDON.

THE researches which we have made in connexion with cancer have convinced us: (1) that we have in cancerous cachexia a specific micro-organism, the micrococcus neoformans of Doyen; (2) that the anti-cancerous sera of Doyen with which we have experimented are wholly useless; and (3) that it is practicable to immunise the human organism by means of a series of inoculations of the micrococcus neoformans vaccine provided that these are properly controlled by examinations of the opsonic power of the blood.

In connexion with the first of these subject matters it will suffice to recall to mind that those bacteriologists who, like Metchnikoff, have sought in the different forms of neoplasia for the micrococcus neoformans by the technique prescribed by Doyen have succeeded in finding the micro-organisms, the cultures obtained in the different laboratories being all identical. We ourselves have cultivated the micro organism from 90 per cent. of the tumours examined. Furthermore we have succeeded in producing localised or generalised neoplasic lesions in 30 per cent. of the cases by inoculating young and vigorous cultures of the micro-organism into mice and white rats. Lastly, as a final argument we shall prove in the course of this paper that the micrococcus neoformans is without doubt the agent of cancerous cachexia.

With regard to the serum of Doyen the clinical results were negative, as our experimental researches made on laboratory animals had led us to expect. This serum was destitute of bactericidal, bacteriolytic, agglutinating, or opsonic power. This serum, had it really possessed the requisite qualities, would have been a precious instrument in the hands of the therapeutist for raising rapidly the defensive power of the cachectic individual; it ought to be discarded.

The vaccines, on the contrary, can give results in careful hands but their inoculation should be made under constant control if it is desired to avoid the bad results which we soon found out when using them. In fact, the vaccines of Doyen are unstandardised vaccines. In view of the principles of immunisation as expounded by Wright we grasped one after another the "why" of the variations, of the mortifications or the negative results obtained by the inoculation of doses of vaccine in the rule-of-thumb manner as advocated by Doyen.

To avoid these accidents we propose the employment of vaccine made from cultures of the same age, sterilised at $60^{\circ} \mathrm{C}$., washed freely, and standardised by the enumeration of the bacilli as advocated by Wright. The antibacterial reaction, which is the method of protection of the body, must be closely watched after inoculation. The method which we have employed is based on the plan of defence employed

1 Synopsis of a paper communicated to the Royal Belgian Academy Medicine on Jan. 27th, 1906. 